\title{
Study of Circular Distance in Graphs
}

\author{
Peruri Lakshmi Narayana Varma ${ }^{a}$, and Janagam Veeranjaneyulu ${ }^{\mathrm{b}}$ \\ a,b \\ Division of Mathematics, Department of S \& H, Vignan's Foundation For \\ Science, Technology \& Research, Vadlamudi - 522213, Guntur, India
}

Article History: Received: 11 January 2021; Accepted: 27 February 2021; Published online: 5 April 2021

\begin{abstract}
Circular distance between vertices of a graph has a significant role, which is defined as summation of detour distance and geodesic distance. Attention is paid, this is metric on the set of all vertices of graph and it plays an important role in graph theory. Some bounds have been carried out for circular distance in terms of pendent vertices of graph. Some results and properties have been found for circular distance for some classes of graphs and applied this distance to Cartesian product of graphs $(P) \_2 \times C \_n$. Including $\llbracket P \rrbracket \_2 \times C \_n$, some graphs acted as a circular self-centered. Using this circular distance there exists some relations between various radii and diameters in path graphs. The possible applications were briefly discussed.
\end{abstract}

Keywords: distance, detour distance, circular distance, circular radius, circular diameter Mathematics Subject Classification: $05 \mathrm{C} 12$.

\section{Introduction}

Due to the broad expansion of networks in graph theory, the distance concept has become very important. It has been recognized that the distance concept in graph theory is also useful in software development. Using this distance concept some basic graph theory parameters were defined such as eccentricity, radius, diameter and metric dimension. The above parameters are related to one another and some bounds have been derived for the radius and diameter [1]. The geodesic distance $d(x, y)$ between two vertices $x$ and $y$ in a graph $H$ is the length of a shortest $x-y$ path in $H$. In a graph two vertices $x$ and $y$ are adjacent if and only if $d(x, y)$ is unity and two different vertices $x$ and $y$ are non neighboring if and only if $d(x, y) \geq 2$. The length of the longest $x-y$ path between two vertices $x$ and $y$ is called detour distance $D(x, y)$. The two distances $d(x, y)$ and $D(x, y)$ are metrics on the set of all vertices of $H$. Different results on detour distance were found and compared to geodesic distance [2 - 4].

Sometimes calculating degree of each vertex of a graph $H$ is also important. In that case in addition to the length of the path, consider degree of each vertex in that path. So many real life problems were solved using this graph distance. For example, when a van is traveling to deliver the goods, it has to stop at one point and deliver the goods: consider the point as vertex and the number of goods delivered as degree of that vertex. Similarly it has to travel and deliver the goods at all delivery points, by taking it as a detour $D$-distance and return to home place, taking this distance as $D$-distance. By doing this type of work, it helps to save time and fuel usage [5, 6].

Now a days wireless network is rapidly growing, in this type of network for example radio, mobile etc., are very useful. Assignment of frequencies to transmitters depend on distance between two transmitters [7 - 9]. On the off chance that $\mathrm{L}$ and $\mathrm{M}$ are two urban areas, at that point for a cab driver the distance between the two urban communities is the genuine distance between the two urban areas. Anyway for a bus transport driver, the distance between similar urban areas is only higher than the typical distance since he needs to cover some significant places in and around the two urban communities to get and drop the travelers. So a bus transport driver needs to locate a briefest defeat that starts from $\mathrm{L}$ and closures at $\mathrm{M}$ and goes through every one of the neighboring spots of $\mathrm{L}$ and M [10-13].

In this paper we study a new distance, namely circular distance, between any two vertices of a graph $H$ by considering summation of detour distance and geodesic distance and work on its properties

The circular distance takes part in a vital role in logistical management. For instance, a postman wants to distribute the letters from the post office which is, say, located at a place 'A' to various destinations on the way to another place 'B'. He takes a long trip from A to B so that he can cover as many as places on the way. On the return trip he may select the shortest route to arrive at the post office at the earliest so as to minimize the time, fuel consumption etc. Similarly, in case of delivery of goods from warehouse to various places we may choose longest path and shortest path. This distance was studied in [14]. Further more, this distance is also used in molecular dynamics of physics, crystallography, lattice statistics and physics.

Throughout the article unless otherwise specified we consider undirected, finite, non-trivial, connected graphs without multiple edges and loops. For any unexplained terminology we follow the book [1].

\section{Circular distance and its properties}

Here we explain the concept of circular distance between vertices of a graph:

Definition 2.1. In a connected graph $H, x, y$ are any two vertices, the circular distance between them is defined as summation of detour and geodesic distance. This is represented by $\operatorname{cir}(x, y)$. In otherwords 


$$
\operatorname{cir}(x, y)=\left\{\begin{array}{cc}
D(x, y)+d(x, y) & \text { if } x \neq y \\
0 & \text { if } x=y
\end{array}\right.
$$

Definition 2.2. The circular eccentricity of $y$ is defined as $e^{C}(y)=\max \{\operatorname{cir}(x, y): x \in V(H)\}$.

Definition 2.3. The minimum circular eccentricity of graph $H$ is called circular radius $r^{C}(H)$. The maximum circular eccentricity of graph $H$ is called circular diameter $d^{C}(H)$.

Next, we start with foremost result.

Theorem 2.4. The circular distance is a metric on the set of all vertices of connected graph $H$.

Proof. Let $x, y, z \in V(H)$. Now $\operatorname{cir}(x, y) \geq 0$ and $\operatorname{cir}(x, y)=0$ if and only if $x=y$. Also we have $\operatorname{cir}(x, y)=\operatorname{cir}(y, x)$ graph and hence this distance is symmetric.

Next we will prove that for every $x, y, \operatorname{cir}(x, y)$ contentes the triangular inequality. The detour distance and geodesic distance satisfies triangle inequality (see [2]).

Now $\operatorname{cir}(x, z)=d(x, z)+D(x, z)$ and $\operatorname{cir}(z, y)=d(z, y)+D(z, y)$.Then $\operatorname{cir}(x, z)+\operatorname{cir}(z, y)=$ $(d(x, z)+D(x, z))+(d(z, y)+D(z, y))=(d(x, z)+d(z, y))+(D(x, z)+D(z, y)) \geq d(x, y)+D(x, y) \geq$ $\operatorname{cir}(x, y)$. Thus $\operatorname{cir}(x, z)+\operatorname{cir}(z, y) \geq \operatorname{cir}(x, y)$. Hence the theorem.

In the next theorem, we give bounds for circular distance, in terms of pendent vertices.

Theorem 2.5. If $H$ is a graph having $p+q$ vertices in which $q$ are pendent vertices. Then

$\operatorname{cir}(x, y) \leq \begin{cases}2(p-q+1) & \text { if } x, y \text { are not pendent } \\ 2(p-q+2) & \text { if either } x \text { or } y \text { is pendent } \\ 2(p-q+3) & \text { if both } x \text { and } y \text { are pendent }\end{cases}$

Proof: Let $H$ be a graph with $p+q$ vertices and assume that among them $q$ are pendent vertices. For any two vertices $x, y$ which are not pendent and $x_{1}, x_{2}, \ldots, x_{q}$ be all the pendent vertices. Any $x-y$ path will exclude these vertices and the edges incident with them. Hence $d(x, y) \leq(p-q)$. Similarly $D(x, y) \leq(p-q+2)$. Thus we have $\operatorname{cir}(x, y) \leq 2(p-q+1)$.

Suppose one vertex between $x$ and $y$ (say $x$ ) is a pendent vertex. Then each $x-y$ path will begin at $x$. These paths will go through the edge adjacent with $x$. They will not go through the remaining $q-1$ vertices and edges incident with them. Thus each of these minimum distances will have a maximum of $p-q+1$ edges. Hence $d(x, y) \leq(p-q+1)$. Similarly $D(x, y) \leq(p-q+3)$. Thus we have $\operatorname{cir}(x, y) \leq 2(p-q+2)$.

For pendent vertices $x$ and $y$, the minimal $x-y$ path starts at $x$ and ends at $y$. This minimal path passes through the edges adjacent with both $x$ and $y$ does not go through the rest of $q-2$ pendent vertices. Hence $d(x, y) \leq(p-q+2)$. Similarly $D(x, y) \leq(p-q+4)$. Thus we have $\operatorname{cir}(x, y) \leq 2(p-q+3)$. Hence the theorem.

Theorem 2.6. Let $H$ be a graph. Then we have $r^{C}(H) \leq d^{C}(H) \leq 2 r^{C}(H)$.

Proof: The lower bound is true. By using triangular inequality the upper bound is as shown below.

Let $x, y \in V(H)$ such that circular distance between $x$ and $y$ is equal to circular diameter of $H$. Let $z$ be a vertex of $H$ such that $e^{C}(z)=r^{C}(H)$.

Consider $\quad d^{C}(H)=\operatorname{cir}(x, y) \leq \operatorname{cir}(x, z)+\operatorname{cir}(z, y) \leq e^{C}(z)+e^{C}(z)=2 e^{C}(z)=2 r^{C}(H) . \quad$ Therefore $d^{C}(H) \leq 2 r^{C}(H)$. Hence $r^{C}(H) \leq d^{C}(H) \leq 2 r^{C}(H)$.

Remark 2.7. The above bounds are sharp. For example a cycle graph $C_{n}$, we have $r^{C}\left(C_{n}\right)=d^{C}\left(C_{n}\right)=n$, (see theorem 3.2) and for a path graph $P_{n}$, we have $d^{C}\left(P_{n}\right)=2 r^{C}\left(P_{n}\right)=2(n-1)$, (see theorem 5.4).

Proposition 2.8. Let $x, y \in V(H)$. Then $\left|e^{C}(x)-e^{C}(y)\right| \leq \operatorname{cir}(x, y)$.

Proof: Given two vertices $x, y \in V(H)$, let $z$ be a vertex such that $\operatorname{cir}(x, \mathrm{z})=e^{C}(x)$. Consider $e^{C}(x)=\operatorname{cir}(x, z) \leq \operatorname{cir}(x, y)+\operatorname{cir}(y, z) \leq \operatorname{cir}(x, y)+e^{C}(y) . \quad$ Thus $\quad e^{C}(x)-e^{C}(y) \leq \operatorname{cir}(x, y) . \quad$ Hence $\left|e^{C}(x)-e^{C}(y)\right| \leq \operatorname{cir}(x, y)$. 


\section{Circular distances of some classes of graph}

Here we find the circular diameter and circular radius. We start with the family of complete graphs.

Theorem 3.1. For a complete graph $K_{m}$, where $m \geq 3$. Then the $e^{C}(x)=m$ for all $x \in V(H)$. Thus we have $r^{C}\left(K_{m}\right)=d^{C}\left(K_{m}\right)=m$ and hence $K_{m}$ is circular self-centered.

Proof. In $K_{m}$, the minimum path length between any two vertices is 1 and maximum length of path is $m-1$.

We have $\operatorname{cir}(x, y)=m-1+1=m$. Thus $e^{C}(x)=m$, for all $x \in V(H)$. Therefore $r^{C}\left(K_{m}\right)=d^{C}\left(K_{m}\right)=m$.

Next, we go for class of cycle graph

Theorem 3.2. Let $C_{n}$ be the cycle graph. Then $\operatorname{cir}(x, y)=n$ for every $x_{i}, x_{j}$. Also $r^{C}\left(C_{n}\right)=d^{C}\left(C_{n}\right)=n$ and hence cycle graph is self-centered.

Proof: The vertices of cycle graph $C_{n}$ can be listed in order $x_{1}, x_{2}, \ldots, x_{n}$. Within any two vertices $x_{i}$ and $x_{j}$ there exist two paths. If one path (geodesic path) contain $\alpha$ edges, the other path (longer path) contain $n-\alpha$ edges. Geodesic distance and detour distance is computed along the smallest path and longest path respectively. Thus $\operatorname{cir}\left(x_{i}, x_{j}\right)=\alpha+n-\alpha=n$ for every $x_{i}, x_{j}$. Therefore $e^{C}\left(x_{i}\right)=n$ and $r^{C}\left(C_{n}\right)=d^{C}\left(C_{n}\right)=n$.

Next, we study the wheel graph.

Theorem 3.3. Let $n+1$ be the order of wheel graph $W_{1, n}$. Then $r^{C}\left(W_{1, n}\right)=n+1$ and $d^{C}\left(W_{1, n}\right)=n+2$ for $n \geq 4$. Further we have $r^{C}\left(W_{1,3}\right)=d^{C}\left(W_{1,3}\right)=4$

Proof. In a wheel graph $W_{1, n}$, the vertices are $\left\{x_{0}, x_{1}, \ldots, x_{n}\right\}$. Suppose that $x_{0}$ is adjacent to rest of the vertices.

For $n=3, x_{0}$ is adjacent to $x_{1}, x_{2}, x_{3}$. Then clearly $D\left(x_{i}, x_{j}\right)=3, d\left(x_{i}, x_{j}\right)=1$, for $1 \leq i, j \leq 3$. Thus $\operatorname{cir}\left(x_{i}, x_{j}\right)=4$. Hence the circular eccentricity of every vertex is 4 . Thus $r^{C}\left(W_{l, 3}\right)=d^{C}\left(W_{l, 3}\right)=4$. So $W_{l, 3}$ is self-centered.

For a wheel graph $W_{1, n}$, with $n \geq 4$, we calculate the circular distance between $x_{0}$ and $x_{i}$. We have $d\left(x_{0}, x_{i}\right)=1$ and $D\left(x_{0}, x_{i}\right)=n$. Then $\operatorname{cir}\left(x_{0}, x_{i}\right)=n+1$. Thus $e^{C}\left(x_{0}\right)=n+1$.

Let $x_{i}, x_{j} \in W_{1, n}$, which are adjacent. Thus $D\left(x_{i}, x_{j}\right)=n$ and $d\left(x_{i}, x_{j}\right)=1$. Thus $\operatorname{cir}\left(x_{i}, x_{j}\right)=n+1$

Let $x_{i}, x_{j} \in W_{1, n}$, which are not adjacent. Thus $D\left(x_{i}, x_{j}\right)=n$ and $d\left(x_{i}, x_{j}\right)=2$. Thus $\operatorname{cir}\left(x_{i}, x_{j}\right)=n+2$ Hence $\operatorname{cir}\left(x_{i}, x_{j}\right)= \begin{cases}1+n & \text { if } x_{i}, x_{j} \text { are adjacent } \\ 2+n & \text { if } x_{i}, x_{j} \text { are not adjacent }\end{cases}$

Thus $e^{C}\left(x_{i}\right)=n+2$. This is true for all $x_{i}$. Hence $r^{C}\left(W_{1, n}\right)=n+1$ and $d^{C}\left(W_{1, n}\right)=n+2$.

Further note that the center of $W_{1, n}$ is $\left\{x_{0}\right\}$ and periphery is $\left\{x_{1}, x_{2}, \ldots, x_{n}\right\}$.

Now, we look at complete bipartite graph.

\section{Theorem 3.4.}

1. Let $K_{p, q}$ be the graph of order $p+q$ where $p<q$. Then we have $r^{C}\left(K_{p, q}\right)=2 p$ and $d^{C}\left(K_{p, q}\right)=2 p+2$.

2. For the graph, $K_{p, p}$, we have $r^{C}\left(K_{p, p}\right)=d^{C}\left(K_{p, q}\right)=2 p$ and hence $K_{p, p}$ is self-centered.

Proof. (1) In a graph, $K_{p, q}$, all vertices can be split into two sets $U_{1}$ and $U_{2}$ having $p$ and $q$ vertices respectively.

Let $x_{1}, y_{1} \in U_{1}$. For any $x_{2} \in U_{2}$, we have $x_{1}-x_{2}-y_{1}$ path between $x_{1}$ and $y_{1}$. Thus distance between $x_{1}, y_{1}$ is always 2. and detour distance between $x_{1}, y_{1}$ is always $2(p-1)$. Thus $\operatorname{cir}\left(x_{1}, y_{1}\right)=2+2(p-1)=2 p$. Therefore $\operatorname{cir}\left(x_{1}, y_{1}\right)=2 p \cdots \cdots(1)$.

Similarly for any two vertices $x_{2}, y_{2} \in U_{2}$ we have $\operatorname{cir}\left(x_{2}, y_{2}\right)=2+2 p \cdots \cdots(2)$.

At lost, we determine the the circular distance between a vertex of $U_{1}$ and a vertex of $U_{2}$. 
Let $x_{1} \in U_{1}$ and $y_{2} \in U_{2}$. Then there is path $x_{1}-y_{2}$ in the graph. Then the geodesic distance between $x_{1}, y_{2}$ is always 1 and detour distance between $x_{1}, y_{2}$ is always $2 p-1$. Then $\operatorname{cir}\left(x_{1}, y_{2}\right)=1+2 p-1=2 p$. Therefore $\operatorname{cir}\left(x_{1}, y_{2}\right)=2 p$

From the above three equations, clearly $e^{C}\left(x_{1}\right)=2 p$ and $e^{C}\left(x_{2}\right)=2 p+2$. Thus minimum circular eccentricity is $2 p$ and maximum circular eccentricity is $2 p+2$. Thus $r^{C}\left(K_{p, q}\right)=2 p$ and $d^{C}\left(K_{p, q}\right)=2 p+2$.

Similarly, in the case of $K_{p, p}, r^{C}\left(K_{p, p}\right)=d^{C}\left(K_{p, q}\right)=2 p$.

\section{Cartesian product of the graphs $P_{2}$ and $C_{n}$.}

Here we prove that for all $n$, the Cartesian product of graph $P_{2} \times C_{n}$ is circular self-centered

Theorem 4.1. Let $P_{2} \times C_{n}$ be the product of graph having $2 n$ vertices. Then for $n \geq 3$.

$$
r^{C}\left(P_{2} \times C_{n}\right)=d^{C}\left(P_{2} \times C_{n}\right)=\left\{\begin{array}{cc}
\frac{5 n}{2} & n(\text { even })=4 p \\
\frac{5 n-2}{2} & n(\text { even })=4 p+2 . \\
\frac{5 n-1}{2} & \text { if } n \text { is odd }
\end{array}\right.
$$

Proof. The vertices of the graph $P_{2} \times C_{n}$ can be listed in order as $x_{1}, x_{2}, \cdots, x_{2 n}$.

Now let us calculate the radius and diameter of $P_{2} \times C_{n}$ for $n \geq 3$ using circular distance.

Case (1): Let $n=4$.

Here the circular distances between the vertices of $P_{2} \times C_{4}$ are as shown in table [1].

\begin{tabular}{|c|c|c|c|c|c|c|c|c|}
\hline & $x_{1}$ & $x_{2}$ & $x_{3}$ & $x_{4}$ & $x_{5}$ & $x_{6}$ & $x_{7}$ & $x_{8}$ \\
\hline$x_{1}$ & 0 & 8 & 8 & 8 & 8 & 8 & 10 & 8 \\
\hline$x_{2}$ & 8 & 0 & 8 & 8 & 8 & 8 & 8 & 10 \\
\hline$x_{3}$ & 8 & 8 & 0 & 8 & 10 & 8 & 8 & 8 \\
\hline$x_{4}$ & 8 & 8 & 8 & 0 & 8 & 10 & 8 & 8 \\
\hline$x_{5}$ & 8 & 8 & 10 & 8 & 0 & 8 & 8 & 8 \\
\hline$x_{6}$ & 8 & 8 & 8 & 10 & 8 & 0 & 8 & 8 \\
\hline$x_{7}$ & 10 & 8 & 8 & 8 & 8 & 8 & 0 & 8 \\
\hline$x_{8}$ & 8 & 10 & 8 & 8 & 8 & 8 & 8 & 0 \\
\hline
\end{tabular}

Table 1. Circular distance of $P_{2} \times C_{4}$ graph

From this table1, we can observe that circular eccentricity of each vertex is 10 . Thus this graph is circular selfcentered with $r^{C}\left(P_{2} \times C_{4}\right)=d^{C}\left(P_{2} \times C_{4}\right)=10$. We can also observe that $e(x)+e_{D}(x)=e^{C}(x)$ for all vertices $r$ in this graph.

\begin{tabular}{|c|c|c|c|c|}
\hline & \multicolumn{2}{|c|}{$r^{C}\left(P_{2} \times C_{n}\right)$} & \multicolumn{2}{|c|}{$d^{C}\left(P_{2} \times C_{n}\right)$} \\
\hline$P_{2} \times C_{2}$ & & 4 & & 4 \\
\hline$P_{2} \times C_{4}$ & & 10 & & 10 \\
\hline$P_{2} \times C_{6}$ & & 14 & & 14 \\
\hline$P_{2} \times C_{8}$ & & 20 & & 20 \\
\hline$P_{2} \times C_{10}$ & & 24 & & 24 \\
\hline$\vdots$ & & $\vdots$ & & $\vdots$ \\
\hline$P_{2} \times C_{n}$ & $\left\{\begin{array}{c}\frac{5 n}{2} \\
\frac{5 n-2}{2}\end{array}\right.$ & $\begin{array}{c}\text { if } n=4 p \\
\text { if } n=4 p+2\end{array}$ & $\left\{\begin{array}{c}\frac{5 n}{2} \\
\frac{5 n-2}{2}\end{array}\right.$ & $\begin{array}{c}\text { if } n=4 p \\
\text { if } n=4 p+2\end{array}$ \\
\hline
\end{tabular}

Table2. $r^{C}\left(P_{2} \times C_{n}\right)$ and $d^{C}\left(P_{2} \times C_{n}\right)$ of a graph ( $n$ is even $)$

Similar calculation will lead to the table2, from which we can conclude that 


$$
r^{C}\left(P_{2} \times C_{n}\right)=d^{C}\left(P_{2} \times C_{n}\right)=\left\{\begin{array}{cc}
\frac{5 n}{2} & \text { if } n=4 p \\
\frac{5 n-2}{2} & \text { if } n=4 p+2
\end{array}\right.
$$

Case 2 Let $n=5$.

Here the circular distances between the vertices of $P_{2} \times C_{5}$ are as shown in table 3 .

\begin{tabular}{|c|c|c|c|c|c|c|c|c|c|c|}
\hline & $x_{1}$ & $x_{2}$ & $x_{3}$ & $x_{4}$ & $x_{5}$ & $x_{6}$ & $x_{7}$ & $x_{8}$ & $x_{9}$ & $x_{10}$ \\
\hline$x_{1}$ & 0 & 10 & 11 & 11 & 10 & 10 & 11 & 12 & 12 & 11 \\
\hline$x_{2}$ & 10 & 0 & 10 & 11 & 11 & 11 & 10 & 11 & 12 & 12 \\
\hline$x_{3}$ & 11 & 10 & 0 & 10 & 11 & 12 & 11 & 10 & 11 & 12 \\
\hline$x_{4}$ & 11 & 11 & 10 & 0 & 10 & 12 & 12 & 11 & 10 & 11 \\
\hline$x_{5}$ & 10 & 11 & 11 & 10 & 0 & 11 & 12 & 12 & 11 & 10 \\
\hline$x_{6}$ & 10 & 11 & 12 & 12 & 11 & 0 & 10 & 11 & 11 & 10 \\
\hline$x_{7}$ & 11 & 10 & 11 & 12 & 12 & 10 & 0 & 10 & 11 & 11 \\
\hline$x_{8}$ & 12 & 11 & 10 & 11 & 12 & 11 & 10 & 0 & 10 & 11 \\
\hline$x_{9}$ & 12 & 12 & 11 & 10 & 11 & 11 & 11 & 10 & 0 & 10 \\
\hline$x_{10}$ & 11 & 12 & 12 & 11 & 10 & 10 & 11 & 11 & 10 & 0 \\
\hline
\end{tabular}

From the above table3, we can observe that $e^{C}\left(x_{i}\right)=12$ for every $x_{i}$. Thus the graph is circular self-centered with $r^{C}\left(P_{2} \times C_{5}\right)=d^{C}\left(P_{2} \times C_{5}\right)=12$. We can also observe that $e(x)+e_{D}(x)=e^{C}(x)$ for all vertices of $x$ in this graph.

Similar calculation will lead to the table4 from which we can conclude that $r^{C}\left(P_{2} \times C_{n}\right)=d^{C}\left(P_{2} \times C_{n}\right)=\frac{5 n-1}{2}$

\begin{tabular}{|c|c|c|}
\hline & $r^{C}\left(P_{2} \times C_{n}\right)$ & $d^{C}\left(P_{2} \times C_{n}\right)$ \\
\hline$P_{2} \times C_{3}$ & 7 & 7 \\
\hline$P_{2} \times C_{5}$ & 12 & 12 \\
\hline$P_{2} \times C_{7}$ & 17 & 17 \\
\hline$P_{2} \times C_{9}$ & 22 & 22 \\
\hline$P_{2} \times C_{11}$ & 27 & 27 \\
\hline$\vdots$ & $\vdots$ & $\vdots$ \\
\hline$P_{2} \times C_{n}$ & $\frac{5 n-1}{2}$ & $\frac{5 n-1}{2}$ \\
\hline
\end{tabular}

Table4. $r^{C}\left(P_{2} \times C_{n}\right)$ and $d^{C}\left(P_{2} \times C_{n}\right)$ of a graph $(n$ is odd $)$

Hence the theorem.

\section{Trees with respect to circular distance.}

Here we study the some results on trees.

Theorem 5.1. Let $H$ be the path graph if and only if $\operatorname{cir}(x, y)=2 d(x, y)=2 D(x, y)$ for all vertices $x, y$.

Proof. If $H$ is a path graph, then there exist one and only one path between any two vertices of $H$. Hence the shortest and longest paths are the same. Thus $d(x, y)=D(x, y)$. Then $\operatorname{cir}(x, y)=2 d(x, y)=2 D(x, y)$.

Conversely, $\operatorname{cir}(x, y)=2 d(x, y)=2 D(x, y)$ for all vertices $x, y$.

We have to prove that $H$ is a path graph. If $H$ has two vertices the answer is trivial. Assume that $n \geq 3$. If $H$ is not a path graph then it contains at least one cycle. Let $C$ be a cycle with $m$ vertices, where $3 \leq m \leq n$. Suppose there is an edge between $x, y$ in $H$ which are in $C$. Then $d(x, y)=1, D(x, y)=m-1$. Thus $\operatorname{cir}(x, y)=m$, which is not true because $H$ is a graph such that $\operatorname{cir}(x, y)=.2 d(x, y)=2 D(x, y)$. Thus $H$ must be a path graph.

Below we determine the circular diameter and circular radius of path graph.

Theorem 5.2. Let $P_{n}$ be the path graph of order $n$. Then we have for $n \geq 3$

$$
r^{C}\left(P_{n}\right)=\left\{\begin{array}{cc}
n & \text { if } n \text { is even } \\
n-1 & \text { if } n \text { is odd }
\end{array} \text { and } \quad d^{C}\left(P_{n}\right)=2 n-2 .\right.
$$


Further $r^{C}\left(P_{2}\right)=d^{C}\left(P_{2}\right)=2$

Proof. The vertices of path graph $P_{n}$ can be listed in order $x_{1}, x_{2}, \ldots, x_{n}$ such that the edges are $\left\{x_{i} x_{i+1}\right\}$ where $i=1,2,3, \cdots, n-1$. There is one and only path between two vertices and hence detour distance and geodesic distance are equal.

Now for $n=2, e^{C}\left(x_{i}\right)=2$ for every $x_{i}$. Hence $P_{2}$ is a self-centered graph with $r^{C}\left(P_{2}\right)=d^{C}\left(P_{2}\right)=2$.

Now let us calculate the radius and diameter of $P_{n}$ for $n \geq 3$ using circular distance. This we will do when $\mathrm{n}$ is even and odd.

Case (1): Assume $n$ is even.

Here the circular distances between the vertices of $P_{n}$ are as shown in table 5 .

\begin{tabular}{|c|c|c|l|c|c|c|c|c|c|c|}
\hline & $x_{1}$ & $x_{2}$ & $\ldots$ & $x_{\frac{n}{2}-1}$ & $x_{\frac{n}{2}}$ & $x_{\frac{n}{2}+1}$ & $\ldots$ & $x_{n-1}$ & $x_{n}$ & $e^{C}$ \\
\hline$x_{1}$ & 0 & 2 & $\cdots$ & $n-4$ & $n-2$ & $n$ & $\cdots$ & $2 n-4$ & $2 n-2$ & $2 n-2$ \\
\hline$x_{2}$ & 2 & 0 & $\cdots$ & $n-6$ & $n-4$ & $n-2$ & $\cdots$ & $n+4$ & $2 n-4$ & $2 n-4$ \\
\hline$\vdots$ & $\vdots$ & $\vdots$ & $\vdots$ & $\vdots$ & $\vdots$ & $\vdots$ & $\vdots$ & $\vdots$ & $\vdots$ & $\vdots$ \\
\hline$x_{n}-1$ & $n-4$ & $n-6$ & $\cdots$ & 0 & 2 & 4 & $\cdots$ & $n$ & $n+2$ & $n+2$ \\
\hline$x_{n}$ & $n-2$ & $n-4$ & $\cdots$ & 2 & 0 & 2 & $\cdots$ & $n-2$ & $n$ & $n$ \\
\hline$\frac{2}{2}$ & & & & & & & & & & \\
\hline$\frac{n}{2}+1$ & $n$ & $n-2$ & $\cdots$ & 4 & 2 & 0 & $\cdots$ & $n-4$ & $n-2$ & $n$ \\
\hline$\vdots$ & $\vdots$ & $\vdots$ & $\vdots$ & $\vdots$ & $\vdots$ & $\vdots$ & $\vdots$ & $\vdots$ & $\vdots$ & $\vdots$ \\
\hline$x_{n-1}$ & $2 n-4$ & $n+4$ & $\cdots$ & $n$ & $n-2$ & $n-4$ & $\cdots$ & 0 & 2 & $2 n-4$ \\
\hline$x_{n}$ & $2 n-2$ & $2 n-4$ & $\cdots$ & $n+2$ & $n$ & $n-2$ & $\cdots$ & 2 & 0 & $2 n-2$ \\
\hline
\end{tabular}

Table5. circular distance of path graph when $n$ is even

From the table5, we see that the circular eccentricities of vertices are $\{2 n-2,2 n-4, \cdots, n+2, n, n, n+2, \cdots, 2 n-4,2 n-2\}$. Thus the minimum circular eccentricity is $n$ and maximum is $2 n-2$. Hence the circular radius and diameter are $n, 2 n-2$ respectively, when $n$ is even.

Case (2): Assume $n$ is odd.

\begin{tabular}{|c|c|c|l|c|c|c|c|c|c|c|}
\hline & $x_{1}$ & $x_{2}$ & $\ldots$ & $x_{n-1}$ & $x_{n+1}$ & $x_{n+3}$ & $\ldots$ & $x_{n-1}$ & $x_{n}$ & $e^{C}$ \\
\hline$x_{1}$ & 0 & 2 & $\cdots$ & $n-3$ & $n-1$ & $n+1$ & $\cdots$ & $2 n-4$ & $2 n-2$ & $2 n-2$ \\
\hline$x_{2}$ & 2 & 0 & $\cdots$ & $n-5$ & $n-3$ & $n-1$ & $\cdots$ & $2 n-6$ & $2 n-4$ & $2 n-4$ \\
\hline$\vdots$ & $\vdots$ & $\vdots$ & $\vdots$ & $\vdots$ & $\vdots$ & $\vdots$ & $\vdots$ & $\vdots$ & $\vdots$ & $\vdots$ \\
\hline$x_{\frac{n-1}{2}}$ & $n-3$ & $n-5$ & $\cdots$ & 0 & 2 & 4 & $\cdots$ & $n-1$ & $n+1$ & $n+1$ \\
\hline$x_{\frac{n+1}{2}}$ & $n-1$ & $n-3$ & $\cdots$ & 2 & 0 & 2 & $\cdots$ & $n-3$ & $n-1$ & $n-1$ \\
\hline$x_{\frac{n+3}{2}}$ & $n+1$ & $n-1$ & $\cdots$ & 4 & 2 & 0 & $\cdots$ & $n-5$ & $n-3$ & $n+1$ \\
\hline$\vdots$ & $\vdots$ & $\vdots$ & $\vdots$ & $\vdots$ & $\vdots$ & $\vdots$ & $\vdots$ & $\vdots$ & $\vdots$ & $\vdots$ \\
\hline$x_{n-1}$ & $2 n-4$ & $2 n-6$ & $\cdots$ & $n-1$ & $n-3$ & $n-5$ & $\cdots$ & 0 & 2 & $2 n-4$ \\
\hline$x_{n}$ & $2 n-2$ & $2 n-4$ & $\cdots$ & $n+1$ & $n-1$ & $n-3$ & $\cdots$ & 2 & 0 & $2 n-2$ \\
\hline
\end{tabular}

Table 6. circular distance of path graph when $n$ is odd

From the table 6, we see that the circular eccentricities of vertices are $\{2 n-2,2 n-4, \cdots, n+3, n+1, n-1, n+1, n+3, \cdots, 2 n-4,2 n-2\}$. Thus the minimum circular eccentricity is $n-1$ and maximum is $2 n-2$. and maximum Hence circular radius and diameter are $n-1,2 n-2$, when $n$ is odd.

Remark 5.3: In a path graph $P_{n}$ when $n$ is odd we observe that $d^{C}\left(P_{n}\right)=2 r^{C}\left(P_{n}\right)$.

Theorem 5.4. Let $P_{n}$ be a path graph having $n$ vertices and $n \geq 3$. Then we have

1. if the center of $P_{n}$ consist of a single vertex then $d^{C}\left(P_{n}\right)=2 r^{C}\left(P_{n}\right)$.

2. if the center of $P_{n}$ consist of a two adjacent vertices then $d^{C}\left(P_{n}\right)=2 r^{C}\left(P_{n}\right)-2$. 
Proof. We can demonstrate this hypothesis in two cases in particular when $n$ is even and odd.

If $n$ is even then center of $P_{n}$ consist of two adjacent vertices. From above theorem $d^{C}\left(P_{n}\right)=2 n-2$ and $r^{C}\left(P_{n}\right)=n$. Hence $d^{C}\left(P_{n}\right)=2 r^{C}\left(P_{n}\right)-2$.

If $n$ is odd then center of $P_{n}$ consist of a single vertex. From the above theorem $d^{C}\left(P_{n}\right)=2 n-2$ and $r^{C}\left(P_{n}\right)=n-1$. Hence $d^{C}\left(P_{n}\right)=2 n-2=2(n-1)=2 r^{C}\left(P_{n}\right)$.

Next in a star graph, circular diameter and radius are independent of $n$.

Theorem 5.5. Let $S t_{n, 1}$ be the star graph. Then we $r^{C}\left(S t_{n, 1}\right)=2, d^{C}\left(S t_{n, 1}\right)=4$.

Proof: The vertices of star graph $S t_{n, 1}$ can be listed in order $x_{0}, x_{1}, x_{2}, \ldots, x_{n}$. Suppose that $x_{0}$ is adjacent to all other vertices so that $d\left(x_{0}, x_{i}\right)=D\left(x_{0}, x_{i}\right)=1$. The circular distances between various vertices are as shown in table 7. Using these, We have $\operatorname{cir}\left(x_{0}, x_{i}\right)=2 d\left(x_{0}, x_{i}\right)=2(1 \leq i \leq n) \quad$ and $\operatorname{cir}\left(x_{i}, x_{j}\right)=2 d\left(x_{i}, x_{j}\right)=4(1 \leq i, j \leq n)$.

\begin{tabular}{|c|c|c|c|c|c|c|}
\hline & $x_{0}$ & $x_{1}$ & $x_{2}$ & $\cdots$ & $x_{n}$ & $e^{C}\left(S t_{n, 1}\right)$ \\
\hline$x_{0}$ & 0 & 2 & 2 & $\cdots$ & 2 & 2 \\
\hline$x_{1}$ & 2 & 0 & 4 & $\cdots$ & 4 & 4 \\
\hline$x_{2}$ & 2 & 4 & 0 & $\cdots$ & 4 & 4 \\
\hline$\vdots$ & $\vdots$ & $\vdots$ & $\vdots$ & $\vdots$ & $\vdots$ & $\vdots$ \\
\hline$x_{n}$ & 2 & 4 & 4 & $\cdots$ & 0 & 4 \\
\hline
\end{tabular}

Table 7. circular distance in star graph

It follows that the circular eccentricities are $\{2,4\}$. Hence circular radius is 2 and diameter is 4 . Hence $r^{C}\left(S t_{n, 1}\right)=2, d^{C}\left(S t_{n, 1}\right)=4$ as claimed.

Remark5.6. We observe that in a star graph $d^{C}\left(S t_{n, 1}\right)=2 r^{C}\left(S t_{n, 1}\right)=4 \forall n$.

Theorem 5.7. Let $x, y$ be the two vertices of tree $T$ which are adjacent. Then we have $\left|e^{c}(x)-e^{c}(y)\right| \leq 2$.

Proof: Choose an eccentric vertex $z$ of $x$ such that $\operatorname{cir}(x, z)=e^{c}(x)$. By triangular inequality $\operatorname{cir}(x, z) \leq \operatorname{cir}(x, y)+\operatorname{cir}(y, z) \quad$ implies $\quad e^{c}(x) \leq d(x, y)+D(x, y)+e^{C}(y) \quad$ implies $\quad e^{c}(x)-e^{c}(y) \leq 1+1$

Therefore $\left|e^{C}(x)-e^{C}(y)\right| \leq 2$

Remark5.8: The above theorem may not be true for a graph which is not a tree.

Theorem 5.9. In a tree the circular periphery consists of pendent vertices only.

Proof: Let $T$ be a tree. Then between any two vertices the maximum circular distance occurs then they must be end vertices. Thus if a vertex has maximum circular distance then that vertex must be in the circular periphery. Hence the circular periphery of a tree contains pendent vertices only.

Some of the future applications.

Below we discuss some possible applications of the concept of circular distance in real life. Here we discuss three of such applications.

Delivery of goods using drones .

Now a days some of the delivery companies are using the drones to deliver the goods. The drones will have the option to fly up to 15 miles and convey bundles under five pounds to clients in under $\$ 30 \$$ minutes time. For instance the Amazon prime company used this for the first time. At present it is using the one to one correspondence procedure to deliver goods. In this method it will take more cost and time. To reduce the cost and time we can use this new concept of circular distance. Using this concept, when we feed the delivery points to the system, the drone will choose the detour path containing more delivery points and choose the shortest path to come back.

\section{Automatic map generation of driverless car with safe journey}

In future we will have most updated driverless cars. While going on a tour using these cars, we have to set the destination and visiting places. Then automatically car will go through these places by using some algorithm. Suppose when we use this circular distance concept in these cars, the tour will go smoothly with low cost and can be reduced time also. Considering the vertices as visiting places and giving this information to the car system by using circular distance concept it will generate the map which consists of detour path containing more visiting places and shortest path while coming back home. Thus we will reduce the time and cost of the tour. 


\section{V2V communication in 5G technology}

In driverless vehicles, there must be communication between vehicles. For instance while traveling, generally we come across signals in junctions. Near the signals the car has to stop and communicate to the vehicles behind it, that ahead there is a red signal and reduce the speed of the car. This message has to go to the vehicles behind up to certain number of cars depending upon the programming. Again the destination car has to send signals to the source car that it received the message. Thus all the cars reduce their speed automatically. When the source car communicates the message it will choose the detour path and destination car sends the message using the shortest path. Indirectly here the mechanism is circular distance. Here the cars are vertices. Using this mechanism we can reduce accidents of the cars in traffic and we will have more peaceful and safe journey.

\section{Conclusion}

In this work, we studied circular distance in a graph and we proved that circular distance is metric. We concentrate few properties of circular distance. We found the circular distance of some families of graphs. We proved that few of the graphs are self-centered. Further we proved $P_{2} \times C_{n}$ is also a self-centered graph.

In future work, we want to study radio circular distance between edges of a graph and also find the relation between circular distance and radio circular distance of a graph. Further we will study the properties of radio circular distance of a graph.

\section{References}

1. F. Buckley, F. Harary. Distance in Graphs. Addison-Wesley., Redwood City, 1990.

2. G. Chartrand, H. Escuadro, P. Zhang. Detour distance in graphs. J Combin Math Combin Compute 53:7594, 2005.

3. G. Chartrand, P. Zhang, T. Haynes. Distance in graphs - taking the long view. AKCE International Journal of Graphs and Combinatorics 1(1):1-13, 2004. https://www.tandfonline.com/doi/pdf/10.1080/ 09728600.2004.12088775.

4. W. Goddard, O. R. Oellermann. Distance in graphs. In Structural Analysis of Complex Networks, pp. 4972. BirkhÃd'userBoston, 2010. doi:10.1007/978-0-8176-4789-6_3.

5. D. Reddy Babu, P. L. N. Varma. D- distance in graphs. Golden Research Thoughts 2:53-58, 2013. doi:10.9780/2231-5063/292013/1696.

6. J. Veeranjaneyulu, P. L. N. Varma. Circular d-distance and path graphs. International Journal of Recent Technology and Engineering 7:219-223, 2019.

7. [R. Gil-Pons, Y. Ram Ãyrez-Cruz, R. Trujillo-Rasua, I. G. Yero. Distance-based vertex identification in graphs: The outer multiset dimension. Applied Mathematics and Computation 363:124-612, 2019. doi: https://doi.org/10.1016/j.amc.2019.124612.

8. S. Prabhu, Y. S. Nisha, K. S. Sudhakhar. Detour distance sequence of grid and grid derived networks. International Journal Of Advanced Research in Basic Engineering Sciences and Technology (IJARBEST) 4:62-67, 2018.

9. R. ÄŇada, J. Ekstein, P. Holub, O. Togni. Radio libelings of distance graphs. Discrete Applied Mathematics 161 (18):2876 - 2884, 2013. doi: https://doi.org/10.1016/j.dam.2013.06.024.

10. Santhakumaran, A. P., P. Titus. Monophonic distance in graphs. Discrete Mathematics, Algorithms and Applications 03(02):159-169, 2011. https://doi.org/10.1142/S1793830911001176.

11. K. M. Kathireson, G. Marimuthu. Superior distance in graphs. J .Combin. Math. Combin. Compute 61:73 80, 2007.

12. M. C. Prajapathi. Distance in graph theory and its applications. International Journal of Advanced Engineering Technology II:147-150, 2011.

13. M. I. Huilgol. Distance degree regular graphs and distance degree injective graphs: An overview. Journal of Discrete Mathematics 2014, pp:12, 2014. https://doi.org/10.1155/2014/358792. 116.

14. V. Kaladevi, P. Selvarani. Three polynomials in one matrix. Mathematical Sciences International Research Journal, 1(1):102-110, 2012. 